\title{
Rate of Escape of Some Chaotic Julia Sets
}

\section{Gonzalo Contreras-Barandiarán ${ }^{\star}$}

Instituto de Matemática Pura e Aplicada ${ }^{\star \star}$, Estrada Dona Castorina, 110, 22460, Rio de Janeiro, Brasil

Received February 27, 1990

Abstract. We give a formula for the rates of escape for Julia sets with preperiodic critical points and for $C^{\infty}$ endomorphisms of the interval with non-flat pre-periodic critical points outside the basin of attracting periodic points.

\section{Introduction}

Let $f: M \hookleftarrow$ be a continuous mapping of a riemannian manifold and $U \subseteq M$. The rate of escape of $U$ (by $f$ ) is defined to be

$$
R(U):=\lim _{n \rightarrow+\infty} \frac{1}{n} \log \operatorname{vol}\left(\bigcap_{k=0}^{n-1} f^{-k}(U)\right) \leq 0
$$

if this limit exists, i.e. the exponential decay of the volume of the set of points which stay on $U$ for $n$ iterates. If $f$ is Axiom A and $U$ a small neighbourhood of a basic set $\Lambda$, Bowen and Ruelle [2], [3] proved that

$$
R(U)=P\left(\varphi^{u}\right)=\sup \left\{h_{v}(f)-\Sigma \lambda_{i}^{+}(v) \mid v \text { ergodic measure with } \operatorname{Supp}(v) \subset \Lambda\right\},
$$

where $\lambda_{i}^{+}(v)$ are the positive Lyapunov exponents of $v, P$ is the topological pressure, $\varphi^{u}(x)=-\log |\operatorname{det} D f| E^{u}(x) \mid$ and $E^{u}(x)$ is the unstable space at $x \in \Lambda$. Axiom A attractors are characterized by $P\left(\varphi^{u}\right)=0$, when $\Lambda$ is not an attractor, $P\left(\varphi^{u}\right)=R(U)<0$ give a measure of the influence of $\Lambda$ on neighbouring orbits. Similar methods [12] apply to prove that $R(U)=P(\varphi), \varphi(x)=-\log \left|\operatorname{det} f^{\prime}(x)\right|$ if $U$ is a small neighbourhood of $K$ and $f: K \hookleftarrow$ is strictly expanding.

Eckmann and Ruelle [3] raised the conjecture that for some open set $U \supset \operatorname{Supp}(\mu)$,

$$
R(U)=h_{\mu}(f)-\Sigma \lambda_{i}^{+}(\mu)
$$

\footnotetext{
Research supported by CNPq, Brasil

${ }^{\star \star}$ Telex: 2121145 ; Fax: 5124115
} 
if $\mu$ is an ergodic measure which maximizes the quantity $h_{\nu}(f)-\Sigma \lambda_{i}^{+}(v)$ when $v$ runs over the invariant probabilities with $\operatorname{Supp}(v) \subset U$. It is clear that $U$ can not be arbitrary (see example of Fig. 17, [4]). Young proved this formula when $U$ is a small neighbourhood of $\Lambda$ and $f: \Lambda \hookleftarrow$ is uniformly partially hyperbolic, i.e. there exists a continuous splitting $T_{A} M=E^{u} \oplus E^{c s}$ such that for an iterate $N>0, D f^{N}$ is strictly expanding on $E^{u}$ and not expanding on $E^{c s}$.

If $f: \overline{\mathbb{C}} \hookleftarrow$ is an analytic endomorphism of the riemann sphere $\overline{\mathbb{C}}=\mathbb{C} \cup\{\infty\}$ (i.e. a rational map), then its Julia set $J(f)$ usually acts as a repulsor. It is then natural to ask about escapes of $J(f)$. Let $c(f)$ be the set of critical points of $f$. Here we prove

Theorem A. If $f: \overline{\mathbb{C}} \hookleftarrow$ is a rational map such that the positive orbit of $c(f) \cap J(f)$ is finite then for any open set $U$ such that $\bigcap f^{n}(\bar{U}) \subset \operatorname{int}(U)$, the following limit exists and the formula holds:

$$
\begin{aligned}
R(U) & =R(\bar{U})=\lim _{n \rightarrow+\infty} \frac{1}{n} \log \operatorname{vol}\left(\bigcap_{k=0}^{n-1} f^{-k}(U)\right) \\
& =\min \left\{0, \sup \left\{h_{v}(f)-2 \int \log \left|f^{\prime}\right| d v \mid v \in \mathscr{M}(f), \operatorname{Supp}(v) \subset U\right\}\right\},
\end{aligned}
$$

where $\mathscr{M}(f)$ is the space of $f$-invariant Borel probabilities.

Thus proving the conjecture for Julia sets with pre-periodic critical points. In particular it holds when all the critical are pre-periodic but not periodic and then the Julia set is the whole sphere. If $J(f)$ has a parabolic periodic point then it actually attracts an open set to the parabolic orbits and $R(U)=0$ for any open set $U$ containing one of those orbits.

The only analytical tool that we use on the proof of Theorem A is Sullivan's structure theorem for rational maps. Its analogous for real one dimensional dynamics is a theorem by Martens, de Melo, and van Strien $[9,10]$. We explain it. Let $N$ be $[-1,1]$ or $S^{1}$ and $f: N \rightarrow N$ a $C^{2}$ non-invertible map having a finite number of critical points which are non-flat. If $f$ has some turning points let $\operatorname{Sing}(f)$ be the set of turning points of $f$ together with the boundary points of $N$. If $f$ has no turning point and is not a diffeomorphism, it must be a covering map of the circle and, therefore, it has a fixed point. In this case define $\operatorname{Sing}(f)$ as the set of fixed points of $f$. We define the Julia set $J(f)$ of $f$ as the $\alpha$-limit of $\operatorname{Sing}(f)$, and the Fatou set $F(f)$ as the complement of $J(f)$. In general $F(f)$ is not forward invariant. If $U$ is a connected component of $J(f)$ then $f(U)$ is contained in the closure of some component of $F(f)$. The theorem says that the components of $F(f)$ are eventually periodic and the number of periodic components is finite. Therefore any critical point in $F(f)$ must have $\omega$-limit a periodic orbit in $F(f)$ and the conditions of $f$ in the following theorem are equivalent to say that the $\omega$-limit of every critical point is a periodic orbit. With this remark, the proof of Theorem B is the same as that of Theorem A:

Theorem B. Let $N$ be $[-1,1]$ or $S^{1}$ and $f: N \rightarrow N$ a $C^{\infty}$ non-invertible map with a finite number of critical points all of which are non-flat. If the positive orbit of $c(f) \cap J(f)$ is finite then for any open set $U \subset N$ such that $\bigcap_{n \geq 0} f^{n}(\bar{U}) \subseteq \operatorname{int}(U)$, we have

$$
R(U)=R(\bar{U})=\min \left\{0, \sup \left\{h_{v}(f)-\int \log \left|f^{\prime}\right| d v \mid v \in \mathscr{M}(f), v(U)=1\right\}\right\} .
$$


Another example is the map $f(z)=2-z^{2}$. If we see $f$ as $f:[-2,2] \hookleftarrow$, then $f$ is conjugate to the Tent map $g(x)=2-|x|$. The Lebesgue measure is invariant by $g$, coincides with the maximal entropy measure for $g$ and is sent by the conjugacy to the measure of maximal entropy for $f$, which is absolutely continuous with respect to the Lebesgue measure. Therefore, by Pesin's formula $h_{\mu}(f)=h_{\mathrm{TOP}}(f)=\log 2=\lambda_{\mu}$, and the rate of escape from $\mu$ in $[-2,2]$ is 0 . If we see $f$ as $f: \overline{\mathbb{C}} \hookleftarrow$, then its Julia set $J(f)=[-2,2]$ is the complement of the basin of $\infty$. If $U$ is a small neighbourhood of $[-2,2]$, we have, using Ruelle's inequality and the methods of Theorem $\mathrm{A}$, that

$$
-\log 2 \leq R(U)=\sup \left\{h_{v}(f)-2 \lambda_{v}\right\} \leq-\inf _{\substack{v \in \mathscr{M}(f) \\ v(J(f))=1}} \lambda_{v} \leq-\frac{1}{2} \log 2<0 .
$$

In particular, $J(f)$ acts as an "exponential" repulsor.

\section{The General Inequality}

We shall use a characterization of metric entropy due to Katok. Let $T: K \hookleftarrow$ be a continuous mapping of a compact metric space $(K, d)$ and $\mu$ a $T$-invariant Borel probability. Let $\varrho: K \rightarrow] 0,1]$ be a measurable function such that $\log \varrho \in \mathscr{L}^{1}(\mu)$. For $x \in K$ and an integer $n>0$, let

$$
V(x, n, \varrho):=\left\{y \in K \mid d\left(T^{k}(x), T^{k}(y)\right)<\varrho\left(T^{k}(x)\right), 0 \leq k<n\right\} .
$$

We say that $E \subset K$ is $(n, \varrho)$-separated if $V(x, n, \varrho) \cap V(y, n, \varrho)=\phi$ whenever $x, y \in E$ and $x \neq y$. For $0<\partial<1, \varepsilon>0$ a set $G \subseteq K$ is said $\mu-(n, \varepsilon, \partial)$-spanning if

$$
\mu\left(\bigcup_{x \in G} V(x, n, \varepsilon)\right) \geq 1-\partial
$$

taking $\varrho \equiv \varepsilon$, the constant function; $G \subseteq K$ is said $(n, \varepsilon)$-spanning if

$$
K \subseteq \bigcup_{x \in G} V(x, n, \varepsilon)
$$

Let $N_{T}(n, \varepsilon, \partial)$ be the minimal cardinality of any $\mu-(n, \varepsilon, \partial)$-spanning set.

1.1. Theorem (Katok [7]). If $\mu$ is ergodic then for every $0<\partial<1$ :

$$
\begin{aligned}
h_{\mu}(f) & =\lim _{\varepsilon \rightarrow 0} \liminf _{n \rightarrow+\infty} \frac{1}{n} \log N_{T}(n, \varepsilon, \partial) \\
& =\lim _{\varepsilon \rightarrow 0} \limsup _{n \rightarrow+\infty} \frac{1}{n} \log N_{T}(n, \varepsilon, \partial) .
\end{aligned}
$$

Recall that the capacity of a compact metric space $K$ is defined as

$$
c(K):=\limsup _{r \rightarrow 0} \frac{\log N(r)}{\log (1 / r)},
$$

where $N(r)$ denotes the minimum number of balls of radius $r$ required to cover $K$.

1.2. Theorem (Brin-Katok-Mañé) [8]. Let $T: K \hookleftarrow$ be a continuous map of $a$ compact metric space $K$ with capacity $(K)<+\infty$. Let $\mu$ be a $T$-invariant Borel 
probability measure and $\varrho: K \rightarrow] 0,+\infty]$ a measurable function such that $\log \varrho \in$ $\mathscr{L}^{1}(\mu)$. Let

$$
\begin{aligned}
h_{\mu}^{+}(T, x) & :=-\lim _{\varepsilon \rightarrow 0} \liminf _{n} \frac{1}{n} \log \mu(V(x, n, \varepsilon \varrho)), \\
h_{\mu}^{+}(T, x) & :=-\lim _{\varepsilon \rightarrow 0} \limsup _{n} \frac{1}{n} \log \mu(V(x, n, \varepsilon \varrho)),
\end{aligned}
$$

then $h_{\mu}^{ \pm}(T, T(x))=h_{\mu}^{ \pm}(T, x)$ for $\mu$-almost every $x \in K$, and $h_{\mu}(T)$ $=\int h_{\mu}^{ \pm}(T, x) d \mu(x)$.

Now let $f: \overline{\mathbb{C}} \hookleftarrow$ be a rational map of the Riemann sphere $\overline{\mathbb{C}}=\mathbb{C} \cup\{\infty\}$ and $\mu$ an ergodic $f$-invariant probability measure. Observe that $\log \left|f^{\prime}\right|$ is measurable and bounded from above. Then by Birkhoff's theorem, the unique Lyapunov exponent $\lambda$ of $\mu$ is

$$
\begin{aligned}
\lambda(x) & :=\lim _{n \rightarrow+\infty} \frac{1}{n} \log \left|\left(f^{n}\right)^{\prime}(x)\right|=\lim _{n \rightarrow+\infty} \frac{1}{n} \sum_{j=0}^{n-1} \log \left|f^{\prime}\left(f^{j}(x)\right)\right| \\
& =\int \log \left|f^{\prime}\right| d \mu .
\end{aligned}
$$

By Ruelle's inequality $h_{\mu}(f) \leq 2 \max \{0, \lambda\}$. Now suppose $h_{\mu}(f)>0$, then

$$
0<h_{\mu}(f) \leq 2 \lambda=2 \int \log \left|f^{\prime}\right| d \mu .
$$

Let $c(f)$ be the set of critical points of $f$. Define $\varrho: \overline{\mathbb{C}} \rightarrow] 0,1]$ by

$$
\varrho(z):=\frac{1}{2} \min \{d(z, c(f)), 1\} .
$$

Let us see that $-\log \varrho$ is $\mu$-integrable if $h_{\mu}(f)>0$. It suffices to see that $-\log \varrho$ is $\mu$-integrable on a neighbourhood of each critical point. If $\omega \in c(f)$ and $r>0$ is small enough, $\varrho(z)=\frac{1}{2} d(z, \omega)$ for $z \in B_{r}(\omega)$ and there exist $A>0$ and $m \in \mathbb{Z}^{+}$ such that $\left|f^{\prime}(z)\right| \leq A d(z, \omega)^{m}$ if $z \in B_{r}(z)$. Then $\log \varrho(z)=\log (1 / 2)+\log d(z, \omega)$ and

$$
-\log \varrho(z) \leq \log 2 A-\frac{1}{m} \log \left|f^{\prime}(\omega)\right| .
$$

Since the argument above showed that $\log \left|f^{\prime}\right|$ is $\mu$-integrable when $h_{\mu}(f)>0$, this concludes the proof that $\log \varrho \in \mathscr{L}^{1}(u)$.

The following is a corollary of Koebe's distorsion theorem [6].

1.3. Lemma. For all $\delta>0$, there exists $\varepsilon(\delta)>0$ such that $\varepsilon(\delta) \rightarrow 0$ when $\delta \rightarrow 0$,

$$
\begin{aligned}
e^{-\delta}\left|f^{\prime}(a)\right| d(a, b) & \leq d(f(a), f(b)) \leq e^{\delta}\left|f^{\prime}(a)\right| d(a, b), \\
e^{-\delta}\left|f^{\prime}(a)\right| & \leq\left|f^{\prime}(b)\right| \leq e^{\delta}\left|f^{\prime}(a)\right|
\end{aligned}
$$

for all $a, b \in c(f)$ such that $d(a, b) \leq e(\delta) d(a, c(f))$ and, setting $r=\varepsilon(\delta) d(a, c(f))$, $\left.f\right|_{B_{r}(a)}$ is injective.

Fix $\delta>0$ satisfying $0<\delta<\lambda(\mu)$ and $\varepsilon:=\min \{1, \varepsilon(\delta)\}$, define for $r>0, n \geq 1$

$$
\begin{aligned}
& B_{r}(x):=B(x, r):=\{y \in \overline{\mathbb{C}} \mid d(x, y) \leq r\}, \\
& \alpha_{n}(x):=\min \left\{r \geq 0 \mid V(x, n, \varepsilon \varrho) \subset B_{r}(x)\right\}, \\
& \beta_{n}(x):=\max \left\{r \geq 0 \mid B_{r}(x) \subset V(x, n, \varepsilon \varrho)\right\} .
\end{aligned}
$$



1.4. Proposition (Mañé [8]). (i) If $x \notin \bigcup_{j=0}^{n} f^{-j}(c(f))$ then $\alpha_{n}(x) \leq e^{\delta_{n}}\left|\left(f^{n}\right)^{\prime}(x)\right|^{-1}$ for
$n \geq 1$. Also

$$
e^{\delta_{n}}\left|\left(f^{n}\right)^{\prime}(x)\right| \leq\left|\left(f^{n}\right)^{\prime}(y)\right| \leq e^{\delta_{n}}\left|\left(f^{n}\right)^{\prime}(x)\right|
$$

for all $y \in V(x, n, \varepsilon \varrho)$ and $\left.f^{n}\right|_{V(x, n, \varepsilon \varrho)}$ is 1-1. In particular

$$
\limsup _{n \rightarrow+\infty} \frac{1}{n} \log \alpha_{n}(x) \leq-\lambda(x)
$$

for all $x \notin \bigcup_{j=0}^{\infty} f^{-j}(c(f))$ for which $\lambda(x)$ exists.

(ii) If $\mu$ is an ergodic $f$-invariant Borel probability with $\log \left|f^{\prime}\right| \in \mathscr{L}^{1}(\mu)$ then for $\mu$-a.e. $x$,

$$
\liminf _{n \rightarrow+\infty} \frac{1}{n} \log \beta_{n}(x) \geq-(\lambda+2 \delta) .
$$

1.5. Proposition. Let $f: \overline{\mathbb{C}} \hookleftarrow$ be a rational map and $\mu$ an ergodic $f$-invariant Borel probability measure such that $\log \left|f^{\prime}\right| \in \mathscr{L}^{1}(\mu)$. Then for any open set $U$ with $\mu(U)=1$ we have

$$
h_{\mu}(f)-2 \int \log \left|f^{\prime}\right| d \mu \leq \liminf _{n \rightarrow+\infty} \frac{1}{n} \log m\left(\bigcap_{k=0}^{n-1} f^{-k}(U)\right),
$$

where $m$ is the riemannian measure on $\overline{\mathbb{C}}$.

Proof. Let $\delta>0$ be given. Let $\Lambda=\bigcap_{n \in \mathbf{Z}} f^{-n}(U)$, then $\mu(\Lambda)=1$. Write $\varepsilon=\min \{\varepsilon(\delta), 1\}$ as in 1.3 , then $\varepsilon \rightarrow 0$ when $\delta \rightarrow 0$. By a combination of Ergorov's and Lusin's theorems there exists a compact subset $K \subset A \subset U$ such that $\mu(K) \geq 1-\partial$ for some $0<\partial<1$ and the limit in 1.4 (ii) is uniform on $K$, i.e. there exists a sequence of positive numbers $\varepsilon_{n} \underset{n}{\rightarrow} 0$ such that

$$
\frac{1}{n} \log \beta_{n}(x) \geq-\left(\lambda+2 \delta+\varepsilon_{n}\right)
$$

for all $x \in K$. If $\delta$ is small enough then $\varepsilon<d(K, \overline{\mathbb{C}}-U)$ and $V(x, n, \varepsilon) \subset \bigcap_{k=0}^{n-1} f^{-k}(U)$ for all $x \in K \subset \Lambda$. Let $S_{n} \subset K$ be a maximal $(n, \varepsilon)$-separated set in $K$, then $S_{n}$ is a $\mu-(n, 2 \varepsilon, \partial)$-spanning set because

$$
K \subseteq \bigcup_{x \in S_{n}} V(x, n, \varepsilon) \subseteq \bigcap_{k=0}^{n-1} f^{-k}(U)
$$

We have that $\# S_{n} \geq N(n, 2 \varepsilon, \partial)$ and then

$$
\begin{aligned}
m\left(\bigcap_{k=0}^{n-1} f^{-k}(U)\right) & \geq m\left(\bigcup_{x \in S_{n}} V(x, n, \varepsilon)\right) \geq \sum_{x \in S_{n}} m(V(x, n, \varepsilon)) \\
& \geq \sum_{x \in S_{n}} m(V(x, n, \varepsilon \varrho)) \geq \sum_{x \in S_{n}} Q\left(\beta_{n}(x)\right)^{2} \\
& \geq N(x, 2 \varepsilon, \partial) \exp 2\left(-\lambda+2 \delta+\varepsilon_{n}\right),
\end{aligned}
$$


where $Q>0$ is such that $m\left(B_{r}(x)\right) \geq Q r^{2}$ for all $z \in \overline{\mathbb{C}}$. The proposition follows from taking $\frac{1}{n} \log$, setting $n \rightarrow \infty$ and then $\delta \rightarrow 0$ (and hence $\varepsilon \rightarrow 0$ ).

\section{Large Deviations Argument}

Let $K$ be a compact metric space and $f: K \hookleftarrow$ a continuous map. Denote by $C^{0}(K)$ the space of continuous functions $\varphi: K \rightarrow \mathbb{R}$, with the topology given by the norm $|\varphi|_{0}:=\sup \{|\varphi(x)| \mid x \in K\}$. Let $\mathscr{M}(K)$ be the space of positive Borel measures on $K$ with the weak ${ }^{*}$ topology over $C^{0}(K), \mathscr{P}(K)$ the subspace of Borel probabilities on $K$ and $\mathscr{M}(f)$ the subspace of $f$-invariant Borel probabilities. Write $v(\varphi):=\int \varphi d v$ for $\varphi \in C^{0}(K)$ and $v \in \mathscr{M}(K)$.

2.1. Proposition [5, 14]. Let $f: K \hookleftarrow$ be a continuous map of a compact metric space and $\left\langle m_{n}\right\rangle$ a sequence on $\mathscr{M}(K)$. For $\varphi \in C^{0}(K)$ write

$$
c(\varphi):=\limsup _{n \rightarrow+\infty} \frac{1}{n} \log \int e^{S_{n} \varphi(x)} d m_{n}(x)
$$

where $S_{n} \varphi(x):=\sum_{k=0}^{n-1} \varphi\left(f^{k}(x)\right)$. For $v \in \mathscr{M}(K)$ write

$$
I(v):=\sup \left\{v(\varphi)-c(\varphi) \mid \varphi \in C^{0}(K)\right\}
$$

and for $G \subseteq \mathscr{M}(K)$ write

$$
I(G):=\inf _{v \in G} I(v) .
$$

Then for any compact subset $G \subseteq \mathscr{M}(K)$ we have

$$
\limsup _{n \rightarrow+\infty} \frac{1}{n} \log m\left\{x \in K / \frac{1}{n} \sum_{k=0}^{n-1} \delta_{f^{k_{x}}} \in G\right\},
$$

where $\delta_{x}(A)=1$ if $x \in A, \delta_{x}(A)=0$ if $x \notin A$ for any $x \in K$ and any Borel set $A \subset K$.

For $U \subseteq K, m \in \mathscr{M}(K), \varphi \in C^{0}(K)$ and $x \in K$, let

$$
\begin{aligned}
R^{-}(U) & :=R^{-}(U, f, m):=\liminf _{n \rightarrow+\infty} \frac{1}{n} \log m\left(\bigcap_{k=0}^{n-1} f^{-k}(U)\right), \\
R^{+}(U) & :=R^{+}(U, f, m):=\limsup _{n \rightarrow+\infty} \frac{1}{n} \log m\left(\bigcap_{k=0}^{n-1} f^{-k}(U)\right), \\
U_{n} & :=\bigcap_{k=0}^{n-1} f^{-k}(U), S_{n} \varphi(x):=\sum_{k=0}^{n-1} \varphi\left(f^{k}(x)\right) .
\end{aligned}
$$

2.2. Corollary. If $U \subseteq K$ is compact, and $m \in \mathscr{M}(K)$, then, taking $G_{N}:=\{v \in$ $\left.\mathscr{M}(K) / v(K)=1=v\left(U_{N}\right)\right\}$ and $m_{n}:=\left.m\right|_{U_{n}}$, we have

$$
R^{+}(U) \leq-I\left(G_{N}\right) \text {. }
$$

2.3. Corollary. (a) Let $U \subseteq K$ be compact. Suppose that the entropy map $\mathscr{M}(f) \rightarrow$ $\left[0,+\infty\left[: v \mapsto h_{v}(f)\right.\right.$ is upper semi-continuous on every $v \in \mathscr{M}(f)$ with $\operatorname{Supp}(v) \subset U$. Assume that there exists a continuous function $\phi: U \rightarrow \mathbf{R}$ such that for all $n \geq 1$, 


$$
\begin{aligned}
m(V(x, n, \varepsilon)) \leq & a_{n}(\varepsilon) \exp \left(S_{n} \phi(x)\right) \text { for all } x \in \bigcap_{k=0}^{n-1} f^{-k}(U), \\
& \lim _{\varepsilon \rightarrow 0} \limsup _{n \rightarrow+\infty} \frac{1}{n} \log a_{n}(\varepsilon)=0,
\end{aligned}
$$

and that $\mathscr{M}(f) \cap \mathscr{P}(U) \neq \phi$ and

$$
Q(U):=\sup \left\{h_{v}(f)+\int \phi d v / v(U)=1, v \in \mathscr{M}(f)\right\} \leq 0,
$$

then $R^{+}(U) \leq Q(U)=h_{\mu}(f)+\int \phi d \mu$ for some $\mu \in \mathscr{M}(f) \cap \mathscr{P}(U)$. (b) If $\mathscr{M}(f) \cap \mathscr{P}(U)=\phi$ then there exists $N \geq 1$ such that $\bigcap_{k=0}^{n-1} f^{-k}(U)=\phi$ for all
$n \geq N$ and hence $R^{-}(U)=R^{+}(U)=-\infty$.

Given $\varphi \in C^{0}(K)$, the topological pressure of $\varphi$ is defined as

$$
P(\varphi):=\lim _{\varepsilon \rightarrow 0} \limsup _{n \rightarrow+\infty} \frac{1}{n} \log \inf _{G} \sum_{x \in G} e^{S_{n} \varphi(x)},
$$

where the infimum is taken over all the $(n, \varepsilon)$-spanning sets for $K$. The variational principle [13] says that for any $\varphi \in C^{0}(K)$,

$$
P(\varphi)=\sup \left\{h_{\mu}(f)+\int \varphi d \mu / \mu \in \mathscr{M}(f)\right\} .
$$

Proof of 2.3. For $\varepsilon>0, n \geq 1, \varphi \in C^{0}(K)$, let $G_{n}$ be an $(n, \varepsilon)$-spanning set for $K$ and

$$
k(\varepsilon):=\sup _{d(x, y) \leq \varepsilon}|\varphi(x)-\varphi(y)|+\sup _{d(x, y) \leq \varepsilon}|\phi(x)-\phi(y)|,
$$

then $k(\varepsilon) \rightarrow 0$ when $\varepsilon \rightarrow 0$, and, if $m_{n}:=\left.m\right|_{U_{n}}$,

$$
\begin{aligned}
\int e^{S_{n} \varphi(x)} d m_{n}(x) & =\int_{U_{n}} e^{S_{n} \varphi(x)} d m(x) \\
& \leq \sum_{x \in G_{n} \cap U_{n}} \exp \left(S_{n} \varphi(x)+n k(\varepsilon)\right) m(V(x, n, \varepsilon)) \\
& \leq \sum_{x \in G_{n} \cap U_{n}} a_{n}(\varepsilon) \exp \left(S_{n} \varphi(x)+S_{n} \phi(x)+n k(\varepsilon)\right) \\
& \leq \sum_{x \in G_{n}} a_{n}(\varepsilon) \exp \left(S_{n} \varphi(x)+S_{n} \phi(x)+n k(\varepsilon)\right) .
\end{aligned}
$$

Let $c(\varphi)$ be as in (2.1) then by the definition of topological pressure, for any $\delta>0$ we can choose $0<\varepsilon<\delta$ and $G_{n}$ such that $c(\varphi) \leq P(\varphi+\phi)+k(\varepsilon)+\delta$, and hence $c(\varphi) \leq P(\varphi+\phi)$.

If $v \in \mathscr{M}(f)$ and $v(U)=1$, then

$$
\begin{aligned}
I(v) & :=\sup _{\varphi}(v(\varphi)-c(\varphi)) \geq \sup _{\varphi}(v(\varphi)-P(\varphi+\phi)) \\
& \geq-\inf _{\varphi}[P(\varphi+\phi)-v(\varphi+\phi)]-v(\phi) .
\end{aligned}
$$


Since $v \mapsto h_{v}(f)$ is upper semicontinuous at $v$, then ([13] 9.12), $\inf _{\varphi}[P(\varphi+\phi)-$ $v(\varphi+\phi)]=h_{v}(f)$. Thus

$$
I(v) \geq-h_{v}(f)-v(\phi) \geq-Q(U) \quad \text { for any } \quad v \in \mathscr{M}(f) \cap \mathscr{P}(U) .
$$

If $v \notin \mathscr{M}(f), v \in \mathscr{P}(K)$ then, by Theorem 9.11 of [13], for $\psi=\varphi+\phi$,

$$
I(v) \geq \sup _{\varphi}(v(\psi)-P(\psi))-v(\phi)>-v(\phi), \quad \text { if } \quad v \notin \mathscr{M}(f) .
$$

For $n \geq 1, z \in K$, let $v_{n}(z):=\frac{1}{n} \sum_{k=0}^{n-1} \delta_{f^{k}(z)} \in \mathscr{P}(K)$. For $N \geq 1$ let

$$
G_{N}:=\text { closure of }\left\{v_{n}(z) / v_{n}(z) \in \mathscr{P}(U), n \geq N\right\},
$$

then $G_{N} \subseteq \mathscr{P}(K)$ is compact for all $N \geq 1$ and like in Corollary (2.2), $R^{+}(U) \leq$ $-I\left(G_{N}\right)$ for all $N \geq 1$. But

$$
I\left(G_{N}\right)=\inf _{v \in G_{N}} I(v) \geq \min \left\{-Q(U), \inf \left\{-v(\phi) / v \in G_{n}, v \notin \mathscr{M}(f)\right\}\right\}
$$

for all $N \geq 1$. Hence $R^{+}(U) \leq \max \left\{Q(U), \limsup _{N}\left(b_{N}\right)\right\}$ where $b_{N}:=\sup \{v(\phi) /$ $\left.v \in G_{N}, v \notin \mathscr{M}(f)\right\}$. There exists a subsequence $v_{N}=v_{N}\left(z_{N}\right) \in G_{N}, v_{N} \notin \mathscr{M}(f)$ such that $\lim \sup v_{N}(\phi)=\lim \sup \left(b_{N}\right)$. Since $G_{N} \subset \mathscr{P}(U)$ and $\mathscr{P}(U)$ is compact, there exists a subsequence $\left\langle v_{m}\right\rangle$ of $\left\langle v_{N}\right\rangle$ such that $v_{m} \rightarrow \mu \in \mathscr{P}(U)$. We will see that $\mu \in \mathscr{M}(f) \cap \mathscr{P}(U)$. Indeed, if $\psi \in C^{0}(K)$,

$$
\begin{aligned}
\mu(\psi \circ f) & =\lim _{m} \frac{1}{m} \sum_{k=0}^{m-1} \psi\left(f^{k+1} z_{m}\right) \\
& =\lim _{m} \frac{1}{m} \sum_{k=0}^{m-1} \psi\left(f^{k} z_{m}\right)-\frac{1}{m} \psi\left(z_{m}\right)+\frac{1}{m} \psi\left(f^{m} z_{m}\right) \\
& =\lim _{m} v_{m}(\psi)=\mu(\psi),
\end{aligned}
$$

we have

$$
\underset{N}{\lim \sup } b_{N}=\mu(\phi) \leq h_{\mu}(f)+\mu(\phi) \leq Q(U)
$$

and hence $R^{+}(U) \leq Q(U)$.

(b) Suppose $\bigcap_{k=0}^{\infty} f^{-k}(U) \neq \phi$, then $G_{N} \neq \phi$ for all $N \geq 1$. Since the compacts $G_{N}$ satisfy $G_{N+1}^{k=0} \subseteq G_{N}$, there exists $\mu \in \bigcap_{N} G_{N}$. The argument above shows that $\mu \in \mathscr{M}(f) \cap \mathscr{P}(U)$. If $\mathscr{M}(f) \cap \mathscr{P}(U)=\phi$, then since $\bigcap_{N} U_{N}=\phi$ is an intersection of a decreasing sequence of compact sets, there must be some $N>0$ such that $U_{n}=\phi$ for all $n \geq N$. Thus $R^{+}(U)=-\infty$.

Given a rational map $f: \overline{\mathbb{C}} \hookleftarrow$ denote by $J(f)$ its Julia set (see [1]), Fix $(f):=$ $\{z \in \overline{\mathbb{C}} / f(z)=z\}$ and $\operatorname{Per}(f):=\bigcup_{n \geq 1} \operatorname{Fix}\left(f^{n}\right)$. Let $m \in \mathscr{P}(\overline{\mathbb{C}})$ be the normalized
riemannian measure. 
2.4. Proposition. Let $f: \overline{\mathbb{C}} \hookleftarrow$ be a rational map such that $c(f) \cap J(f) \subseteq$ $\bigcup_{n \geq 1} f^{-n}(\operatorname{Per}(f))$. If $U \subseteq \dot{\overline{\mathbb{C}}}$ is compact, then

$$
R^{+}(U) \leq \sup \left\{h_{v}(f)-2 \int \log \left|f^{\prime}\right| d v / v \in \mathscr{M}(f), v(U)=1\right\} .
$$

We begin with some reductions. If there exists $v \in \mathscr{M}(f), v(U)=1$ with $\log \left|f^{\prime}\right| \notin$ $\mathscr{L}^{1}(v)$ then the lefthand side of the inequality is $+\infty$, since always $R^{+}(U) \leq 0$, (2.4) holds. So assume $\log \left|f^{\prime}\right| \in \mathscr{L}^{1}(v)$ for any $v \in \mathscr{M}(f), v(U)=1$. Observe that it is enough to prove (2.4) when $c(f) \cap J(f) \subseteq \bigcup_{n \geq 1} f^{-n}(\operatorname{Fix}(f))$. Indeed, we can always find an iterate $k \geq 1$ such that for $g=f^{k}, c(g) \cap J(g) \subseteq \bigcup_{n \geq 1} g^{-n}(\operatorname{Fix}(g))$.
Given $Q \geq 1$ let $n \geq 1$ be such that $n k<Q \leq(n+1) k$, then

$$
\begin{aligned}
\frac{1}{n} \log m\left(\bigcap_{i=0}^{n-1} g^{-i}(U)\right) & \leq k \frac{1}{n k} \log m\left(\bigcap_{j=0}^{n k-1} f^{-i}(U)\right) \\
& \geq k \frac{Q}{n k} \frac{1}{Q} \log m\left(\bigcap_{j=0}^{Q-1} f^{-j}(U)\right)
\end{aligned}
$$

Also, for all $\mu \in \mathscr{M}(f)$ sucht that $\log \left|f^{\prime}\right| \in \mathscr{L}^{1}(\mu)$,

$$
\begin{aligned}
h_{v}(g)-2 \int \log \left|f^{\prime}\right| d \mu & =k h_{\mu}(f)-2 \int \sum_{j=0}^{k-1} \log \left|f^{\prime}\right|\left(f^{j}(z)\right) \mid d \mu(z) \\
& =k\left(h_{\mu}(f)-2 \int \log \left|f^{\prime}\right|=d \mu\right) .
\end{aligned}
$$

Suppose (2.4) is true for $g$, then

$$
k\left(h_{\mu}(f)-2 \int \log \left|f^{\prime}\right| d \mu\right) \geq k \frac{Q}{n k} \frac{1}{Q} \log m\left(\bigcap_{j=0}^{Q-1} f^{-j}(U)\right) .
$$

Since $1 \leq \frac{Q}{n k} \leq \frac{n+1}{n} \rightarrow 1$ when $Q \rightarrow+\infty$ we get (2.4) for $f$.

From now on let $f: \overline{\mathbb{C}} \hookleftarrow$ be a rational map with $c(f) \cap J(f) \subset \bigcup_{n \geq 1} f^{-n}(\operatorname{Fix}(f))$. Let $\mathscr{C}(f):=c(f) \cap J(f)$. Observe that there are no singular domains $D$ (Siegel discs or Herman rings) because if so the boundary $\partial D$ must be in the closure of the positive orbits of critical points which are in $J(f)$ (cf. [1]) and $\mathscr{C}(f)$ has finite positive orbit.

Suppose that there exists a parabolic periodic point $p$ in $U$, i.e. $p \in U$ with $f^{n}(p)=p, \lambda:=\left(f^{n}\right)^{\prime}(p), \lambda^{k}=1$ for some $n, k \geq 1$. If $v$ is the ergodic measure supported in the positive orbit of $p$, we have

$$
0 \geq R^{+}(U) \geq R^{-}(U) \geq h_{v}(f)-0=0 .
$$

Suppose that there exists a critical point $\omega \in c(f) \cap J(f)$ such that $f^{n}(\omega)=p$ is a fixed point such that $\left|f^{\prime}(p)\right| \leq 1$. Then $p$ must be a parabolic fixed point and we may assume that $p \notin U$. But then $p \notin U_{n+1}$ and since $R^{+}\left(U_{n+1}\right)=R^{+}(U)$, we can assume that $p \notin U$.

If $\omega \in c(f)$ and in local coordinates $f(z)=f(\omega)+a_{m}(z-\omega)^{m}+\ldots$ for $z$ near $\omega$, write $m:=\operatorname{ord}_{\omega}(f)$. 
2.5. Lemma. If $\omega \in c(f), f^{n}(\omega)=p, f(p)=p, \lambda:=\left|f^{\prime}(p)\right|>1$, then there exist $E=E(\omega)>0, \ell=\ell(\omega) \geq 1, D=D(\omega)>0$ such that for all $R>0$ sufficiently small there are $S=S(R)>0$ and $\varepsilon_{0}>0$ depending on $R$ such that $S(R) \rightarrow 0$ when $R \rightarrow 0$ and

(a) for all $z \in B(\omega, E S)-B(\omega, S), f^{n+\ell}(z) \notin B(p, R)$,

(b) $\left.f^{n}(B \omega, S)\right) \subset B(p, R)$,

(c) for all $z \in B(\omega, E S), z \neq p$ and $0<\varepsilon \leq \varepsilon_{0}$; if $q>0$ is the first integer $q \in \mathbb{Z}^{+}$ such that

$$
R<d\left(f^{n+q}(z), p\right) \leq 2\left|f^{\prime}(p)\right| R,
$$

then

$$
\begin{aligned}
V(z, n+q, \varepsilon) & \subset V\left(z, n+q, \varepsilon D R^{-1} \varrho\right), \\
\operatorname{diam} V(z, n+1, \varepsilon) & \leq D R^{\frac{m-1}{m}} \lambda^{q / m} \varepsilon, \\
D^{-1} R^{\frac{m-1}{m}} \lambda^{q / m} & \leq\left|\left(f^{n+q}\right)^{\prime}(z)\right| \leq D R^{\frac{m-1}{m}} \lambda^{q / m} \\
D^{-1} R^{\frac{m-1}{m}} \lambda^{-q \frac{(m-1)}{m}} & \leq\left|\left(f^{n}\right)^{\prime}(z)\right| \leq D R^{\frac{m-1}{m}} \lambda^{-q\left(\frac{m-1}{m}\right)} .
\end{aligned}
$$

Proof. Choose $S_{0}<0$ and $0<b<1$ such that for all $x \in B\left(\omega, S_{0}\right)$,

$$
\begin{aligned}
b d(x, \omega)^{m} & \leq d\left(f^{n}(x), p\right) \leq b^{-1} d(x, \omega)^{m} \\
b d(x, \omega)^{m-1} & \leq\left|\left(f^{n}\right)^{\prime}(x)\right| \leq b^{-1} d(x, \omega)^{m-1} .
\end{aligned}
$$

There exist $R_{0}, T_{0}>0$ and an analytic conjugacy (cf. [1]) $F: B\left(p, R_{0}\right) \rightarrow B\left(0, T_{0}\right)$ such that $F(p)=0, F^{\prime}(p)=1$ and if $x, f(x) \in B\left(p, R_{0}\right), F(f(x))=a F(x)$, where $a:=f^{\prime}(p)$. Choose $A>0$ such that for all $x, y \in B\left(p, R_{0}\right)$ :

$$
\begin{aligned}
A^{-1} d(x, y) & \leq d(F(x), F(y)) \leq A d(x, y) \\
A^{-1} & \leq\left|F^{\prime}(x)\right| \leq A .
\end{aligned}
$$

Take $0<R_{1}<R_{0}$ such that $\frac{1}{2}\left|f^{\prime}(p)\right| \leq\left|f^{\prime}(x)\right| \leq 2\left|f^{\prime}(p)\right|$ for all $x \in B\left(p, R_{1}\right)$. Take $\varepsilon_{0}, R_{2}, S_{2}>0$ such that $0<\varepsilon_{0}<S_{2}<R_{2}<R_{1}<1, f^{n+1}\left(B\left(\omega, 2 S_{2}\right)\right) \subset B\left(p, R_{2}\right)$, $f\left(B\left(p, 2 R_{2}\right)\right) \subset B\left(p, R_{1} / 2\right)$ and

$$
\begin{aligned}
\Delta & :=d\left(B\left(p, R_{0}\right), c(f)\right)>0, \\
d(c, z(f)) & =d(z, \omega) \text { for all } z \in B\left(\omega, 2 S_{2}\right) .
\end{aligned}
$$

Let $R_{3}>0$ be such that $B\left(p, R_{3}\right) \subset f^{n}\left(B\left(\omega, S_{2}\right)\right)$. Suppose $0<R<R_{3}$, let $z \in B\left(\omega, S_{1}\right)$ be such that $f^{n}(z) \in B(p, R)$. Let $q>0$ be the first $q \in \mathbb{Z}^{+}$such that

$$
R<d\left(f^{n+q}(z), p\right) \leq 2\left|f^{\prime}(p)\right| R .
$$

For $x \in V(z, n+q, \varepsilon), 0<\varepsilon<\varepsilon_{0}$, let $x_{n+k}:=f^{n+k}(x), k=0,1, \ldots, q$; then

$$
\begin{aligned}
d\left(x_{n+q}, p\right) & \geq A^{-1} d\left(F\left(f^{-q}\left(x_{n}\right)\right), 0\right) \geq A^{-1} d\left(a^{q} F\left(x_{n}\right), 0\right) \\
& \geq A^{-1} \lambda^{q} d\left(F\left(x_{n}\right), 0\right) \geq A^{-2} \lambda^{q} d\left(x_{n}, p\right) \\
d\left(x_{n}, p\right) & \leq A^{2} \lambda^{-q} d\left(x_{n+q}, p\right)
\end{aligned}
$$

and similarly

$$
d\left(x_{n}, p\right) \geq A^{-2} \lambda^{-q} d\left(x_{n+q}, p\right) .
$$


By (1), we have

$$
\begin{aligned}
& d(x, \omega) \geq b^{1 / m} d\left(x_{n}, p\right)^{1 / m} \geq b^{1 / m} A^{-2 / m} \lambda^{-q / m} d\left(x_{n+q}, p\right)^{1 / m}, \\
& d(x, \omega) \geq \lambda^{-q / m} R^{1 / m} Q^{-1}, \\
& d(x, \omega) \leq \lambda^{-q / m} R^{1 / m} Q
\end{aligned}
$$

where $Q>0$ is such that $0<Q^{-1} \leq\left(b A^{-2} \frac{1}{2}\right)^{1 / m} \leq\left(A^{2} b^{-1} 4\left|f^{\prime}(p)\right|\right)^{1 / m} \leq Q$ and $\frac{1}{2} R<R-\varepsilon<2\left|f^{\prime}(p)\right| R+\varepsilon<4\left|f^{\prime}(p)\right| R$. If we take $\ell>0$ such that $\lambda^{-\ell} 2\left|f^{\prime}(p)\right| A^{2} b^{-1}<b$ then for $E>1, S>0$, such that $\left(2\left|f^{\prime}(p)\right| R \lambda^{-\ell} A^{2} b^{-1}\right)<S<$ $E S<R^{1 / m} b^{1 / m}$ we have that $f^{n}(B(\omega, E S)) \subset B(p, R) \forall x \in B(\omega, E S)-B(\omega, S)$ : $f^{n+\ell}(x) \notin B(p, R)$ and $S(R)=S(\omega, R) \rightarrow 0$ when $R \rightarrow 0$. Using the conjugacy, $F$, we have

$$
\begin{aligned}
\left|\left(f^{n+q}\right)^{\prime}(x)\right| & =\left|\left(f^{n}\right)^{\prime}(x)\right|\left|\left(f^{q}\right)^{\prime}\left(f^{n} x\right)\right| \\
& \geq b d(x, \omega)^{m-1} A^{-2} \lambda^{q} \\
& \geq b Q^{-(m-1)} R^{\frac{m-1}{m}} \lambda^{-q} \lambda^{q / m} A^{-2} \lambda^{q} \\
& \geq D_{0}^{-1} R^{\frac{m-1}{m}} \lambda^{q / m} \\
\left|\left(f^{n+q}\right)^{\prime}\right| & \leq D_{0} R^{\frac{m-1}{m}} \lambda^{q / m}
\end{aligned}
$$

for any $D_{0}>0$ such that $0<D_{0}^{-1}<b Q^{-(m-1)} A^{-2}$. Using the mean value theorem, for all $x \in V(z, n+q, \varepsilon)$ there exists $\eta \in V(z, n+q, \varepsilon)$ such that

$$
\varepsilon \geq d\left(z_{n+q}, x_{n+q}\right)=\left|\left(f^{n+q}\right)^{\prime}(\eta)\right| d(z, x) \geq D_{0}^{-1} R^{\frac{m-1}{m}} \lambda^{q / m} d(z, x) .
$$

By (4), we have

$$
\begin{gathered}
d(x, z) \leq D_{0} \varepsilon R^{-1} R^{1 / m} \lambda^{-q / m} \leq D_{0} Q \in R^{-1} d(z, \omega), \\
d(x, z) \leq D_{1} R^{-1} \varepsilon d(z, \omega),
\end{gathered}
$$

for $D_{1}>D_{0} Q$. By (3), for $k=0,1, \ldots, q$,

$$
d\left(x_{n+k}, z_{n+k}\right) \leq \varepsilon \leq \varepsilon\left(\frac{1}{\Delta}\right) \Delta \leq \varepsilon\left(\frac{1}{\Delta}\right) d\left(z_{n+k}, c(f)\right) .
$$

Therefore, if $D:=\max \left\{\frac{2}{\Delta}, D_{0}, 2 D_{1}\right\}, z \in B\left(\omega, S_{2}\right)$ and $0<\varepsilon<\varepsilon_{0}<1$, we have from (5) that diam $V(z, n+q, \varepsilon) \leq D_{0} \varepsilon R^{-\frac{m-1}{m}} \lambda^{-q / m}$ and from (6) and (7) that $V(z, n+q, \varepsilon) \subset V\left(z, n+q, \varepsilon R^{-1} \varrho\right)$. This completes the proof. We can also take

$$
D \geq \max \left\{1,\left(b^{-1} A^{2}\right)^{1 / m}\right\} .
$$

We want to construct a continuous map $\phi$ which, integrated by ergodic measures, nearly realizes the Lyapunov exponents. Let $\mathscr{C}(f):=c(f) \cap J(f) \cap U$. For $\omega \in \mathscr{C}(f)$ let $n(\omega)$ be the first integer $n(\omega) \in \mathbb{Z}^{+}$such that $p(\omega):=f^{n(\omega)}(\omega) \in \operatorname{Fix}(f)$. We can assume that $\lambda(\omega):=\left|f^{\prime}(p(\omega))\right|>1$ for all $\omega \in \mathscr{C}(f)$. Let $M:=\max \{n(\omega) / \omega \in$ $\mathscr{C}(f)\}$, then $g:=f^{M}$ has the property that in the positive orbit of each $\omega \in \mathscr{C}(f)$ there is at most another critical point in $\mathscr{C}(f)$. Therefore we can assume that $f$ has this property.

We want to separate orbits of pre-periodic points which do not pass through a critical point. Let $M$ be the branched manifold defined as follows. Let $Q(f):=$ 
$\left\{\omega \in \mathscr{C}(f) /\left(f^{n(\omega)}\right)^{\prime}(f(\omega))=0\right\}$. For each $\omega \in Q(f)$ let $V(\omega)$ a small disc centered at $\omega$ such that $f$ is strictly expanding on $f^{n(\omega)}(V(\omega))$ and all the $f^{k}(V(\omega))$, $0 \leq k \leq n(\omega), \omega \in Q(f)$ form a collection of disjoint topological discs. Let $M$ be the quotient space $M:=\tilde{M} / \equiv$, where:

$$
\tilde{M}:=\left(\overline{\mathbf{C}} \times\{0\} \cup \bigcup_{\omega \in Q(f)} \bigcup_{k=1}^{n(\omega)} f^{k}(V(\omega)) \times\{1\}\right)
$$

and $(x, 0) \equiv(y, 1)$ iff $x=y \in \partial f^{k}(V(\omega))$ for some $1 \leq k \leq n(\omega)$ and $\omega \in Q(f)$. Define $g: M \rightarrow M$ by $g(x, 0)=(f(x), 0)$ if $x \notin V(\omega)$ for any $\omega \in Q(f) ; g(x, 0)=$ $(f(x), 1)$ if $x \in V(\omega) ; g(x, 1)=(f(x), 1)$ if $f(x) \in f^{n(\omega)}(V(\omega)) \cup \bigcup_{k=1}^{n(\omega)-1} f^{k}(V(\omega))$ and $g(x, 1)=(f(x), 0)$ if $f(x) \notin f^{n(\omega)}(V(\omega)), x \in f^{n(\omega)}(V(\omega))$. The condition that $f$ is strictly expanding on $f^{n(\omega)}(V(\omega))$ implies that $f$ is continuous and $C^{\infty}$. Let $\pi: M \rightarrow \overline{\mathbb{C}}$ be the canonical projection.

In (2.5) we can choose $\ell, E, D$ uniformly for all $\omega \in \mathscr{C}(f)$, i.e. $\ell \geq \ell(\omega)$, $E \geq E(\omega), D \geq D(\omega)$, for all $\omega \in \mathscr{C}(f)$. Let $\delta_{0}>0$ be so small that all $B\left(f^{k}(\omega), \delta_{0}\right) \quad 0 \leq k \leq n(\omega), \omega \in \mathscr{C}(f)$ are disjoint and $B\left(f^{k}(\omega), \delta_{0}\right) \subset f^{k}(V(\omega))$ for all $0 \leq k \leq n(\omega), \omega \in Q(f)$. Fix $0<\delta<\delta_{0} / 4$ and let $\phi=\phi_{\delta}, \varphi=\varphi_{\delta}$ : $M \rightarrow \mathbb{R}$ be continuous functions defined as follows. Let $0<R \ll \delta$ be such that $\left.f^{3 \ell}(B(p(\omega), R)) \subset B(p(\omega)), \delta\right)$. Let $\omega \in \mathscr{C}(f)-Q(f)$, i.e. $\left(f^{n(\omega)}\right)^{\prime}(f \omega) \neq 0$. Let

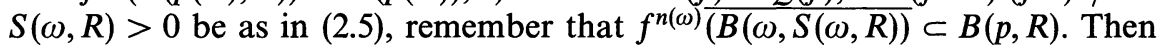

$$
\begin{aligned}
& \phi(z, 0)=-\frac{2}{m(\omega)} \log \lambda(\omega) \quad \text { for } \quad z \in \bigcap_{k=0}^{\ell+2} f^{-k}(B(p(\omega), R)), \\
& -\frac{2}{m(\omega)} \log \lambda(\omega) \leq \phi(z, 0) \leq-2 \log \left|f^{\prime}(z)\right| \quad \text { for } z \in \bigcap_{k=0}^{\ell+1} f^{-k}(B(p(\omega), R)), \\
& \phi(z, 0)=-2 \log \left|f^{\prime}(z)\right| \quad \text { for } \quad z \in B(p(\omega), \delta)-\bigcap_{k=0}^{\ell+1} f^{-k}(B(p(\omega), R)), \\
& \phi(z, 0)=-2 \log D+2 \frac{m(\omega)-1}{m(\omega)} \log R \\
& +\sum_{k=1}^{n(\omega)-1} 2 \log \left|f^{\prime}\left(f^{k} \omega\right)\right| \quad \text { for } \quad z \in B(\omega, S(\omega, R)) \text {, } \\
& \left\{\begin{array}{l}
\phi(z, 0) \geq-2 \log D+2 \frac{m(\omega)-1}{m(\omega)} \log R+\sum_{k=1}^{n(\omega)} 2 \log \left|f^{\prime}\left(f^{k} \omega\right)\right| \\
\text { and } \\
\phi(z, 0) \leq-2 \log \left|f^{\prime}(z)\right| \\
\quad \text { for } z \in\left(f^{-n(\omega)}(B(p(\omega), R))-B(\omega, S(\omega, R))\right) \cap B(\omega, \delta),
\end{array}\right\} \\
& \phi(z, 0)=-2 \log \left|f^{\prime}(z)\right| \text { for } z \in B(\omega, \delta)-f^{-n(\omega)}(B(p(\omega), R)) \text {, }
\end{aligned}
$$




$$
\phi(z, 0)=-2 \log \left|f^{\prime}(z)\right| \text { for } z \in \bigcup_{k=1}^{n(\omega)-1} f^{k}(B(\omega, \delta))
$$

Note that there is a constant $K=K(\omega)>0$ such that $\left|f^{\prime}(z)\right| \leq K(\omega) \lambda^{-\ell / m} R^{\frac{m-1}{m}}$ for all $z \in B(\omega, \delta) \cap f^{-n(\omega)}(B(p(\omega), R))$ so that $(*)$ makes sense if one chooses $D>0$ sufficiently large but independent of $\omega, \delta$ or $R$. Suppose that $z \in B(\omega, S(\omega, R))$ and $q \geq 0$ is the first $q \in \mathbb{Z}^{+}$such that $f^{n(\omega)+q}(z) \notin B(p, R)$, let $n=n(\omega)$, $m=m(\omega), S_{k} \phi(z, 0)=\sum_{i=0}^{k-1} \phi\left(g^{i}(x, 0)\right)$, then, by $(2.5)$,

$$
\begin{aligned}
S_{n+q} \phi(z, 0) & \geq-2 \log D R^{\frac{m-1}{m}} \lambda^{\frac{q-\ell-2}{m}} \lambda^{\ell+2} \\
& \geq-2 \log \left|\left(f^{n+q}\right)^{\prime}(z)\right|-2 \log \lambda^{\ell+2}-4 \log D, \\
S_{n+q} \phi(z, 0) & \geq-2 \log D R^{\frac{m-1}{m}} \lambda^{\frac{q-\ell-1}{m}} \lambda^{\ell+1} \\
& \geq-2 \log D R^{\frac{m-1}{m}} \lambda^{q / m} \\
& \geq-2 \log \left|\left(f^{n+q}\right)^{\prime}(z)\right| .
\end{aligned}
$$

If $z \in\left(f^{-n(\omega)}(B(p(\omega), R))-B(\omega, S(\omega, R))\right) \cap B(\omega, \delta)$ and $q>0$ is the first $q \in$ $\mathbb{Z}^{+}$such that $f^{n(\omega)+q}(z) \notin B(\omega, R)$, then $0<q \leq \ell$ and hence $\phi\left(g^{n+k}(z, 0)\right)=$ $-2 \log \left|f^{\prime}\left(f^{n+k}(z)\right)\right|$ for $0 \leq k \leq q$. We have, by $(2.5)$,

$$
\begin{aligned}
S_{n+q} \phi(z, 0) & \geq-2 \log D R^{\frac{m-1}{m}}-2 \log \left|\left(f^{q}\right)^{\prime}\left(f^{n} z\right)\right| \\
& \leq-2 \log \left|\left(f^{n}\right)^{\prime}(z)\right|-2 \log D^{2} \lambda^{q\left(\frac{m-1}{m}\right)}-2 \log \left|\left(f^{q}\right)^{\prime}\left(f^{n} z\right)\right| \\
& \geq-2 \log \left|\left(f^{n+q}\right)^{\prime}(z)\right|-2 \log D^{2} \lambda^{\ell}, \\
S_{n+q} \phi(z, 0) & \leq-2 \log \left|\left(f^{n+q}\right)(z)\right| .
\end{aligned}
$$

If $z \in B(\omega, \delta)-f^{-n(\omega)}(B(p(\omega), R))$ and $q>0$ is the first $q \in \mathbb{Z}^{+}$such that $f^{n(\omega)+q}(z) \notin B(\omega, R)$, then

$$
S_{n+q} \phi(z, 0)=-2 \log \left|\left(f^{n+q}\right)^{\prime}(z)\right| .
$$

Now suppose that $\omega \in Q(f)$, then we can assume that there exists one and only one critical point in the forward orbit of $\omega$. Let $f^{r}(\omega)=u \in \mathscr{C}(f)$. Define

$$
\begin{gathered}
\phi(z, 1)=\phi(z, 0) \text { for } z \in \bigcup_{k=1}^{n(\omega)-1} f^{k}(V(\omega)), \\
\phi(z, 1)=-\frac{2}{m(\omega)} \log \lambda(\omega) \text { for } z \in \bigcap_{k=0}^{\ell+2}(B(p(\omega), R)), \\
-\frac{2}{m(\omega)} \log \lambda(\omega) \leq \phi(z, 1) \leq-2 \log \left|f^{\prime}(z)\right| \text { for } z \in \bigcap_{k=0}^{\ell+1} f^{-k}(B(p(\omega), R)), \\
\phi(z, 1)=-2 \log \lambda(\omega) \text { for } z \in f^{n(\omega)}(V(\omega))-\bigcap_{k=0}^{\ell+1} f^{-k}(B(p(\omega), R)) .
\end{gathered}
$$


Let $z \in \bigcup_{k=1}^{n(\omega)} f^{k}(V(\omega))$ and let $q \geq 0$ be the first $q \in \mathbb{Z}^{+}$such that $f^{s(z)+q}(z) \notin$ $B(p(\omega), R)$, with $z \in f^{n(\omega)-s(z)} V(\omega)$. Then, by the same arguments as in [9] and [10], we get

$$
\left.S_{n} \phi(z, 1) \geq-2 \log \mid\left(f^{s(z)+q}\right)^{\prime}(z)\right)-2 \log D^{2} \lambda^{\ell+2} .
$$

But we don't get a good upper bound for $S_{n} \phi(z, 1)+2 \log \left|\left(f^{s(z)+q}\right)^{\prime}(z)\right|$ because $\phi$ is $\log \lambda^{-2 / m(\omega)}$ and not $\log \lambda^{-2 / m(u)}$ near $p(\omega)=p(u)$. Let

$$
\begin{gathered}
\phi(z, 0)=-2 \log D-S_{n(\omega)-1} \phi(f(z), 1)-2 \frac{m(\omega)-1}{m(\omega)} \log R \\
\quad \text { for } z \in f^{-(n(\omega)-n(u))}(B(u, S(u, R))) \cap V(\omega), \\
\left\{\begin{array}{l}
\phi(z, 0) \geq-2 \log D-S_{n(\omega)-1} \phi(f(z), 1)-2 \frac{m(\omega)-1}{m(\omega)} \log R \\
\phi(z, 0) \leq-2 \log \left|f^{\prime}(z)\right| \text { for } z \in f^{-n(\omega)}(B(p(\omega), R))
\end{array}\right\}, \\
\phi(z, 0)=-2 \log \left|f^{\prime}(z)\right| \text { for } z \in V(\omega)-f^{-n(\omega)}(B(p(\omega), R)) .
\end{gathered}
$$

If $\delta_{0}$ is small enough, then $S_{n(\omega)-1} \phi(f(z), 1) \geq 0$ and by the same remark as in $(*)$ we can choose a uniform $D$, not depending on $\omega, \delta, R$; such that $(* *)$ can be satisfied by a continuous $\phi$. By the same arguments as above, if $z \in V(\omega)$ and $q>0$ is the first integer such that $f^{n(\omega)+q}(z) \notin B(p(\omega), R)$, we have

$$
\begin{aligned}
& S_{n} \phi(z, 0) \geq-2 \log \left|\left(f^{n(\omega)+q}\right)^{\prime}(z)\right|-2 \log D^{2} \lambda^{\ell+2}, \\
& S_{n} \phi(z, 0) \geq-2 \log \left|\left(f^{n(\omega)+q}\right)^{\prime}(z)\right| .
\end{aligned}
$$

Once $\phi$ is defined on a neighbourhood of all the critical points $\mathscr{C}_{M}(g):=$ $Q(f) \times\{0\} \cup(\mathscr{C}(f)-Q(f)) \times\{0,1\}$, define $\phi$ on the remaining points of $V:=\pi^{-1}(U)$ by

$$
\phi(z, 0)=-2 \log \left|f^{\prime}(z)\right| .
$$

Define $\varphi=\varphi_{\delta}: M \rightarrow \mathbb{R}$ to be a continuous function such that

$$
\begin{aligned}
\varphi(z, \alpha) \leq 2 \log D^{2} \Lambda^{\ell+2} & \text { if } \quad d\left((z, \alpha), \mathscr{C}_{m}(g)\right) \leq \delta, \\
0 \leq \varphi(z, \alpha) \leq 2 \log D^{2} \Lambda^{\ell+2} & \text { if } \delta \leq d\left((z, \alpha), \mathscr{C}_{M}(g)\right) \leq 2 \delta, \\
\varphi(z, \alpha)=0 & \text { if } \quad d\left((z, \alpha), \mathscr{C}_{M}(g)\right)>2 \delta
\end{aligned}
$$

for $\Lambda \geq \max \{\lambda(\omega) / \omega \in \mathscr{C}(f)\}$ and $\alpha=0,1$.

For $\omega \in \mathscr{C}_{M}(g)$ we can define $p(\omega), n(\omega), m(\omega)$ and $\lambda(\omega)$ as we did for $f$. If $z \in B(\omega, \delta) . z \neq \omega \in M$ for some $\omega \in \mathscr{C}_{M}(g)$ and $q>0$ is the first $q \in \mathbb{Z}^{+}$such that $d\left(g^{n(\omega)+q}(z), p(\omega)\right)>R$, we say that the orbit $g^{k}(z), k=0,1, \ldots, n(\omega)+q$ is bound to $(\omega, p(\omega))$ and that it frees at $g^{n(\omega)+q}(z)$. We also say that its forward orbit remains free until it enters to a bound period again.

We have proven the following.

2.6. Lemma. Let $V:=\pi^{-1}(U)$. There exists $A>0$ such that for all $\delta>0$ sufficiently small there are continuous functions $\phi=\phi_{\delta}, \varphi=\varphi_{\delta}: V \rightarrow \mathbb{R}$ such that $0 \leq \varphi(z, \alpha) \leq A$ for all $(z, \alpha) \in V$ and $\varphi(z, \alpha)=0$ if $d(z, \mathscr{C}(f))>2 \delta$ and for all $n \geq 1$

$$
S_{n}(\phi+\varphi)(z, \alpha) \geq-2 \log \left|\left(f^{n}\right)^{\prime}(z)\right|
$$


for all $(z, \alpha) \geq \bigcap_{k=0}^{n} g^{-k}(V)$ such that $g^{n}(z, \alpha)$ is free, and

$$
S_{n} \phi(z, 0) \leq-2 \log \left|\left(f^{n}\right)^{\prime}(z)\right|
$$

for all $z \in \overline{\mathbb{C}}$ such that $z \in \bigcap_{k=0}^{n} f^{-k}(U)$.

2.7. Lemma. There exist $Q>0$ and $\varepsilon_{1}=\varepsilon_{1}(\delta)>0$ such that if $c(f)-J(f) \subset \overline{\mathbb{C}}-U$, $0<\varepsilon<\varepsilon_{1}(\delta), N>0, V:=\pi^{-1}(U)$ and $z \in V_{N}:=\bigcap_{i=0}^{N-1} g^{-i}(V) \subset M$, we have (a) If $g^{N}(z)$ is free $\left(\right.$ i.e. $\left.g^{N}(z) \notin \underset{\substack{\omega \in \mathscr{C}_{M}(g) \\ n(\omega)<N}}{\bigcup_{j=0}^{n(\omega)-1}} g^{-j}(B(p(\omega), R))\right)$ then

$$
m(V(z, N, \varepsilon)) \leq Q \exp \left(4 \delta N+S_{N}(\phi+\varphi)(z)\right) .
$$

(b) If $\omega \in \mathscr{C}_{M}(g), n:=n(\omega), g^{N}(z) \in B(p(\omega, R)), N \geq n(\omega)+k, g^{N-n-k}(z) \in$ $B(\omega, \delta)$ is free, $g^{N}(z)$ is bound to $(\omega, p(\omega))$ and

$$
A_{k}:=\left\{x \in M / g^{N-n-k}(x) \in B(\omega, \delta), f^{N-k+i}(z) \in B(p(\omega), R), \quad \forall i=0,1, \ldots, k\right\},
$$

then

$$
m\left(A_{k} \cap V(z, N, \varepsilon)\right) \leq Q \exp \left(4 \delta N+S_{N}(\phi+\varphi)(z)\right)
$$

and hence

$$
m(V(z, N, \varepsilon)) \leq Q N \exp \left(4 \delta N+S_{N}(\phi+\varphi)(z)\right) .
$$

(c) If $g^{N-k}(z) \in B(\omega, \delta)$ is free, $\omega \in \mathscr{C}_{M}(g)$ and $0 \leq k \leq n(\omega)$, then

$$
m(V(z, N, \varepsilon)) \leq m(V(z, N-k, \varepsilon)) \leq Q \exp \left(4 \delta(N-k)+S_{N-k}(\phi+\varphi)(z)\right),
$$

where $m$ is the riemannian measure on $M, S_{N} \phi(z):=\sum_{i=0}^{N-1} \phi(f(z)), \phi=\phi_{\delta}, \varphi=\varphi_{\delta}$ are from (2.6) and $V(z, N, \varepsilon)=\bigcap_{i=0}^{N-1} g^{-i}\left(B\left(g^{i}(z), \varepsilon\right)\right)$.

Proof. Let $\sigma=\sigma(\delta)>0$ be so small that $g^{n(\omega)+2 \ell}(B(\omega, \sigma)) \subseteq B(p(\omega), R)$ for all $\omega \in \mathscr{C}_{M}(g)$. Let $\varepsilon_{1}(\delta):=\frac{1}{2} \varepsilon(\delta) \min \left\{1, d(c(f), \partial U), D^{-1} R^{2}, \delta, \sigma\right\}$, where $\varepsilon(\delta)$ is from (1.3). Let $Q>0$ be such that for all $x \in M$ and $r>0$,

$$
m(B(x, 2 r)) \leq \frac{1}{2} Q \min \left\{1, D^{-2}\right\}(\operatorname{diam}(B(x, r)))^{2} .
$$

(a) If $g^{N}(z)$ is free, then by (2.5) and the condition $\varepsilon<\min \{\varepsilon(\delta) R, \varepsilon(\delta) \delta, \varepsilon(\delta) \sigma\}$ we have

$$
V(z, N, \varepsilon) \subseteq V(z, N, \eta \varrho) \subseteq V(z, N, \varepsilon(\delta) \varrho),
$$

where $\eta:=\max \left\{\frac{\varepsilon}{\delta}, \frac{\varepsilon}{R}, \frac{\varepsilon}{\sigma}, \frac{D \varepsilon}{R}\right\}$ and $\varrho(z):=\min \left\{1, d\left(z, \mathscr{C}_{M}(g)\right)\right\}$. Also

$$
\operatorname{diam}(V(z, N, \varepsilon)) \leq e^{\delta N}\left|\left(g^{N}\right)^{\prime}(z)\right|^{-1} \leq e^{\delta N} \exp \left(S_{N}(\phi+\varphi)(z)\right),
$$

therefore

$$
m(V(z, N, \varepsilon)) \leq Q \exp \left(2 \delta N+S_{N}(\phi+\varphi)(z)\right) .
$$

(b) Since $g^{N-n-k}(z)$ is free we have that $V(z, N, \varepsilon) \subseteq V(z, N-n-k, \varepsilon) \subseteq V(z, N-$ $n-k, \varepsilon(\delta) \varrho)$. Hence, by (1.3), for $t:=N-n-k, f^{t}: V(z, N-n-k, \varepsilon(\delta) \varrho) \rightarrow B(\omega, \delta)$ 
is 1-1 and, by (2.6), $\left|\left(g^{t}\right)^{\prime}(x)\right| \geq \exp \left(-\delta t-\frac{1}{2} S_{t}(\phi+\varphi)(z)\right)$ for all $x \in V(z, N-n-$ $k, \varepsilon(\delta) \varrho)$. Let

$$
B_{k}:=B(\omega, \delta) \cap \bigcap_{i=0}^{k} g^{-n-i}(B(p(\omega), R))
$$

Then, by the same arguments as in Lemma (2.5) and (8) in (2.5), we have

$$
\begin{gathered}
\operatorname{diam}\left(B_{k}\right) \leq\left(b^{-1} A^{2} R\right)^{1 / m} \lambda^{-k / m} \leq D \lambda^{-k / m} \\
m\left(A_{k} \cap V(z, N, \varepsilon)\right) \exp \left(-\delta t-\frac{1}{2} S_{t}(\phi+\varphi)(z)\right) \leq \int_{A_{k} \cap V(z, N, \varepsilon)}\left|\left(g^{t}\right)^{\prime}(x)\right| d m(x) \\
\leq m\left(B_{k}\right) \leq Q_{0} \lambda^{-2 k / m(\omega)}
\end{gathered}
$$

If $g^{t}(z) \in B(\omega, \sigma)$, then the choice of $\varepsilon_{1}(\delta)$ implies that $V(z, N, \varepsilon) \subset V\left(z, N, \varepsilon_{1}(\delta) \varrho\right)$ and using (1.3) we get in particular (b). Suppose $g^{t}(z) \in B(\omega, \sigma)$, then by the definition of $\varphi, \phi$ and $\sigma>0, \exp \left(S_{n+k}(\phi+\varphi)(z)\right) \geq \lambda(\omega)^{2 k / m(\omega)}$, and hence

$$
\begin{aligned}
m\left(A_{k} \cap V(z, N, \varepsilon)\right) & \geq Q \exp \left(2 \delta t+S_{N}(\phi+\varphi)(z)\right) \\
& \geq Q \exp \left(2 \delta N+S_{N}(\phi+\varphi)(z)\right) .
\end{aligned}
$$

For (c) just aply (a).

2.8. Corollary. There exists $Q>0$ such that if $V:=\pi^{-1}(U), \varepsilon, \phi$ and $\varphi$ are as in Lemma (2.7) then for any $N>0$ and $z \in V_{N}:=\bigcap_{i=0}^{N-1} g^{-i}(V)$, we have

$$
m(V(z, N, \varepsilon)) \leq Q N \exp \left(4 \delta N+S_{N}(\phi+\varphi)(z)\right) .
$$

Proof. Consider the case (c) of (2.7). If $\left(g^{n(\omega)}\right)^{\prime}(g(\omega)) \neq 0$ and $\delta_{0}>0$ is small enough, then $S_{k}(\phi+\varphi)(x) \geq 0$ for all $0 \leq k \leq n(\omega)$ and $x \in B\left(\omega, \delta_{0}\right)$. Hence $S_{N}(\phi+\varphi)(z) \geq S_{N-k}(\phi+\varphi)(z)$ and the inequality holds. If $\left(g^{n(\omega)}\right)^{\prime}(g(\omega))=0$, $g^{r}(\omega)=u \in \mathscr{C}_{M}(g)$, then $-\frac{m(\omega)-1}{m(\omega)} \log R>-\frac{m(u)-1}{m(u)} \log R$ and

$$
\begin{aligned}
\phi(x) \geq & -\left(\frac{m(\omega)-1}{m(\omega)}-\frac{m(u)-1}{m(u)}\right) \log R-2 \log D \\
& +\sum_{\substack{i=1 \\
i \neq n(\omega)-n(u)}}^{n(\omega)-1} 2 \log \left|f^{\prime}\left(f^{i} z\right)\right| .
\end{aligned}
$$

Thus if $\delta_{0}>0$ is small enough $R \ll \delta_{0}$ and $S_{k}(\phi+\varphi)(x) \geq 0$ for $0 \leq k \leq n(\omega)$ and $x \in B\left(\omega, \delta_{0}\right)$, and then $S_{N}(\phi+\varphi)(z) \geq S_{N-k}(\phi+\varphi)(z)$ This complettes the proof.

2.9. Theorem. (Yomdim, Newhouse [11, 15]). Let $M$ be a compact differentiable manifold and $f: M \hookleftarrow$ a map of class $C^{\infty}$. Then the entropy map $\mathscr{M}(f) \rightarrow[0,+\infty[$, $v \mapsto h_{\nu}(f)$ is upper semi-continuous.

2.10. Corollary. For $V:=\pi^{-1}(U)$,

$$
R^{+}(V, g) \leq \liminf _{\delta \rightarrow 0} \sup \left\{h_{v}(g)+v\left(\phi_{\delta}+\varphi_{\delta}\right) / v \in \mathscr{M}(g) \cap \mathscr{P}(V)\right\} .
$$


Proof. By (2.3) and (2.8) we only need to see that the entropy map $v \mapsto h_{v}(g)$ is u.s.c. By (2.9) for $f: \overline{\mathbb{C}} \hookleftarrow, \mu \mapsto h_{\mu}(f)$ is u.s.c. For $v \in \mathscr{M}(g)$, let $\mu=\pi^{*}(v)$ be defined by $\mu(\psi):=v(\psi \circ \pi)$ for any $\psi \in C^{0}(M)$. Let $v \in \mathscr{M}(g)$ be ergodic but $v \neq \delta_{p}$ for any $p \in \mathbf{P}(g):=\left\{p(\omega) / \omega \in \mathscr{C}_{M}(g)\right\}$. Then $\mu=\pi^{*} v$ is ergodic and for $\eta>0$ small,

$$
v\left\{(x, 0) \in M / d\left(x, \bigcup_{\omega \in Q(f)} \bigcup_{i=0}^{n(\omega)} f^{i}(V(\omega))\right)>2 \eta\right\}>0 .
$$

Pick $(x, 0)$ in this set. Then $\pi$ is $1-1$ on $B((x, 0), \eta)$ and

$$
v(V(x, 0), g, n, \varepsilon))=\left(\pi^{*} v\right)(V(x, f, n, \varepsilon))
$$

for any $0<\varepsilon<\eta$. By Brin-Katok's theorem for a set of positive measure of such $(x, 0)$, we have

$$
\begin{aligned}
h_{v}(g) & =\lim _{n \rightarrow+\infty}-\frac{1}{n} \log v(V((x, 0), g, n, \varepsilon)) \\
& =\lim _{n \rightarrow+\infty}-\frac{1}{n} \log \left(\left(\pi^{*} v\right)(V(x, f, n, \varepsilon))\right. \\
& =h_{\pi^{*} v}(f) .
\end{aligned}
$$

For the other ergodic measures in $\mathscr{M}(g), v=\delta_{p(\omega)}, \omega \in \mathscr{C}_{M}(g)$, we have that $h_{v}(g)=0=h_{\pi^{*} v}(f)$. So that $h_{v}(g)=h_{\pi^{*} v}(f)$ for all $v \in \mathscr{M}_{\operatorname{erg}}(g)$ and since the entropy map is affine (cf. [13]), $h_{v}(g)=h_{\pi^{*} v}(f)$ for all $v \in \mathscr{M}(g)$. Let $\left\langle v_{n}\right\rangle \subset \mathscr{M}(g)$, $v_{n} \rightarrow \bar{v}$, then $\mu_{n}:=\pi^{*} v_{n} \rightarrow \pi^{*} v=: \mu$ and $\lim \sup h_{v_{n}}(g)=\lim \sup h_{\mu_{n}}(f) \leq$ $h_{\mu}(f)=h_{v}(g)$.

Proof of Proposition (2.4). We can assume that $c(f)-J(f) \subset \overline{\mathbb{C}}-U$ because all the invariant measures $\mu \in \mathscr{M}(f)$ on $\overline{\mathbb{C}}-J(f)$ have $h_{\mu}(f)=0$ and $-2 \int \log \left|f^{\prime}\right| d \mu=0$ or $+\infty$, and $R^{+}(U, f) \leq 0$. Let $m$ be the riemannian measure on $\overline{\mathbb{C}}$ and $\bar{m}$ be the riemannian measure on $M$, then $m(A) \leq \bar{m}\left(\pi^{-1}(A)\right) \leq 2 m(A)$ for any Borel set $A \subset \overline{\mathbb{C}}$. Hence $R^{+}(U, f, m)=R^{+}(U, g, \bar{m})$.

Let $v \in \mathscr{M}(g)$ be ergodic and $v \neq \delta_{p(\omega)}$ for all $\omega \in \mathscr{C}_{M}(g)$. Then by Birkhoff's theorem there exist $y \in M-\mathscr{C}_{M}(g)$ such that $v\left(\phi_{\delta}\right)=\lim _{n \rightarrow+\infty} \frac{1}{n} S_{n} \phi_{\delta}(y)$ and $v(\bar{\psi})=\lim _{n \rightarrow+\infty} \frac{1}{n} S_{n} \bar{\psi}(y)$, where $\bar{\psi}(z, \alpha)=-2 \log \left|f^{\prime}(z)\right|$. Let $N>0$ be the first time that $z:=f^{N}(y)$ frees and $\pi(z)$ is outside $\bigcup_{\omega \in \mathscr{C}_{M}} f^{n(\omega)}(V(\omega))$. Then $z=(x, 0)$ and $v\left(\phi_{\delta}\right)=\lim _{n \rightarrow+\infty} \frac{1}{n} S_{n} \phi_{\delta}(x, 0)$. If $g^{n}(x, 0)$ is free then, by $(2.6), S_{n} \phi_{\delta}(x, 0)=S_{n} \bar{\psi}(x, 0)$ and since the orbit of $(x, 0)$ must have infinitely many free periods we have $v\left(\phi_{\delta}\right)=v(\bar{\psi})=v(\psi \circ \pi)=\left(\pi^{*} v\right)(\psi)$, where $\psi(z)=-2 \log \left|f^{\prime}(z)\right|$, for $z \in \overline{\mathbb{C}}$. For $v=\delta_{p(\omega)}, \omega \in \mathscr{C}_{M}(g), v\left(\phi_{\delta}\right)=-\frac{2}{m(\omega)} \log \lambda(\omega)$. Since the entropy map is affine we can take the supremum in (2.10) only over ergodic measures. On (2.10) we proved that $h_{v}(g)=h_{\pi^{*} v}(f)$, thus by $(2.6)$

$$
\begin{aligned}
& \sup \left\{h_{v}(g)+v\left(\phi_{\delta}+\varphi_{\delta}\right) / v \in \mathscr{M}(f) \cap \mathscr{P}(V)\right\} \\
& \quad \leq \max \left\{Q(U),-\frac{2}{m(\omega)} \log \lambda(\omega) / \omega \in \mathscr{C}(f)\right\}+A e(\delta),
\end{aligned}
$$


where

$$
\begin{aligned}
Q(U) & :=\sup \left\{h_{\mu}(f)-2 \int \log \left|f^{\prime}\right| d \mu / \mu \in \mathscr{M}(f) \cap \mathscr{P}(U)\right\}, \\
e(\delta) & :=\sup \{v(\mathbf{B}(2 \delta)) / \nu \in \mathscr{M}(f) \cap \mathscr{P}(U)\}, \\
\mathbf{B}(2 \delta) & :=\bigcup_{\omega \in \mathscr{C}_{M}(g)} B(\omega, 2 \delta) .
\end{aligned}
$$

Now we see that $\liminf _{\delta \rightarrow 0} e(\delta)=0$. If not, there exist sequences $\delta_{n} \downarrow 0, v_{n} \rightarrow v$ in $\mathscr{M}(f) \cap \mathscr{P}(U)$ such that $v_{n}\left(\mathbf{B}\left(2 \delta_{n}\right)\right)>a>0$ for all $n \geq 1$. Fix $N \geq 1$ and let $2 \delta_{N+1}<$ $\varepsilon_{N} \leq 2 \delta_{N}$ be such that $v\left(\partial \mathbf{B}\left(\varepsilon_{N}\right)\right)=0$, then $v\left(\mathbf{B}\left(\varepsilon_{N}\right)\right) \leq \liminf v_{n}\left(\mathbf{B}\left(2 \delta_{n}\right)\right) \geq a>0$ for all $N \geq 1$. But when $N \rightarrow 0, \mathbf{B}\left(\varepsilon_{N}\right) \downarrow \mathscr{C}_{M}(g)$ and $v\left(\mathscr{C}_{M}(g)\right)=0$ because otherwise the whole negative orbit of some critical point must have infinite measure. This leads to a contradiction. Therefore

$$
R^{+}(U, f) \leq \max \left\{Q(U),-\frac{2}{m(\omega)} \log \lambda(\omega) / \omega \in \mathscr{C}(f)\right\} .
$$

Let $\omega \in \mathscr{C}(f)$, since $\omega \in J(f)$ its negative orbit is dense in $J(f)$. Thus there exist $z_{0} \in B(p(\omega), \delta)$ and $N>0$ such that $f^{N}\left(z_{0}\right)=\omega$. Take $\varepsilon>0$ such that $B\left(z_{0}, \varepsilon\right) \subset$ $B\left(p(\omega), \delta_{0}\right), f^{N}\left(B\left(z_{0}, \varepsilon\right)\right) \subset B\left(\omega, \delta_{0}\right), f^{2}\left(B\left(z_{0}, \varepsilon\right)\right)>\left|z_{0}\right|$. Given $M>0$ take $n>M$ and $x_{M} \in B\left(p(\omega), \delta_{0}\right)$ such that $f^{n}\left(x_{M}\right)=z_{0}$. Let $y_{M}:=f^{-n(\omega)}\left(x_{M}\right) \cap B\left(\omega, \delta_{0}\right)$. Then $x_{M} \rightarrow p(\omega), y_{M} \rightarrow \omega$ when $M \rightarrow+\infty$ and if $M$ is sufficiently large, $f^{-n-n(\omega)}\left(B\left(z_{0}, \varepsilon\right)\right) \subset f^{N}\left(B\left(z_{0}, \varepsilon\right)\right) \ni \omega$ is a topological ball which is sent into $f^{N}\left(B\left(z_{0}, \varepsilon\right)\right)$ by $f^{n+n(\omega)+N}$. Then there exists a fixed point $z_{M}$ for $f^{n+n(\omega)+N}$ in $B\left(\omega, \delta_{0}\right)$. Let $v_{M}$ be the ergodic measure supported on the orbit of $z_{M}$. Then $h_{v}(f)=0$ and by $(2.5)$

$$
-2 \log \int \log \left|f^{\prime}\right| d v_{M} \geq-\frac{2}{n+n(\omega)+M} \log \left(D\left|z_{0}\right|^{\frac{m-1}{m}} \lambda(\omega)^{\frac{n+2}{n}} A^{N-2}\right)^{2} .
$$

where $m:=m(\omega)$ and $A:=\sup \left\{\left|f^{\prime}(z)\right| / z \in \overline{\mathbb{C}}\right\}$. Since $n=n(M) \rightarrow+\infty$ when $M \rightarrow+\infty, \sup \left\{-2 \int \log \left|f^{\prime}\right| d v_{M} / M \geq 1\right\} \geq-\frac{2}{m(\omega)} \log \lambda(\omega)$. Since $\omega \in \mathscr{C}(f)$ is arbitrary, $Q(U) \geq-\frac{2}{m(\omega)} \log \lambda(\omega)$ for any $\omega \in \mathscr{C}(f)$ and hence $R^{+}(U, f)$ $\leq Q(U)$.

Proof of Theorem A. Let $v \in \mathscr{M}(f)$ be ergodic and $\operatorname{Supp}(v) \subset J(f)$. The arguments in (2.5) prove that for all $x \in J(f)-\bigcup_{k=0}^{\infty} f^{-k}(c(f))$,

$$
\lambda^{-}(x):=\liminf _{n \rightarrow+\infty} \frac{1}{n} \log \left|\left(f^{n}\right)^{\prime}(x)\right|>0 .
$$

Since $\log \left|f^{\prime}\right|$ is bounded from above, by Birkhoff's theorem $\lambda^{-}(x)=\int \log \left|f^{\prime}\right| d v$ $>0$ for $v$-a.e. $x$ and hence $\log \left|f^{\prime}\right| \in \mathscr{L}^{1}(v)$. If $\operatorname{Supp}(v) \subset \overline{\mathbb{C}}-J(f)$ and $\log \left|f^{\prime}\right| \notin$ $\mathscr{L}^{1}(v)$ then $v$ must be supported on the orbit of a periodic critical point $\omega \in c(f)$. If orbit $(\omega) \subset U$, then $R(U)=0$. If $\log \left|f^{\prime}\right| \in \mathscr{L}^{1}(v)$ then since we only need to consider ergodic measures, get from (1.5) one inequality of Theorem A.

If $U$ does not contain critical points in $\overline{\mathbb{C}}-J(f)$ then apply $(2.4)$ and get the other inequality of Theorem A. If $\omega \in c(f) \cap(\overline{\mathbb{C}}-J(f)) \cap U \neq \phi$ then the $\omega$-limit of $\omega$ is a parabolic or attracting periodic orbit $\mathcal{O}(p)$. If $\mathcal{O}(p) \subset \bar{U}$ then $\mathcal{O}(p) \subset U$ and $R(U)=0$. If $\mathcal{O}(p) \subset \bar{U}$ then there exists $N>0$ such that $f^{N}(\omega) \notin \bar{U}$. Then 
$\omega \notin \bar{U}_{N}:=\bigcap_{k=0}^{N} f^{-k}(\bar{U})$. Since $R(\bar{U})=R\left(\bar{U}_{N}\right)$, we can assume $\omega \notin U$ and since the number of critical points of $f$ is finite, we can neglect this case.

Acknowledgements. I want to thank Marcelo Viana and Miguel Paternain for their encouragement and also the hospitality of ICTP, Trieste and Centro de Estudos Matemáticos do Porto where part of this research was done.

\section{References}

1. Blanchard, P.: Bull. Am. Math. Soc. (New Series) 11-1, 85-141

2. Bowen, R.: Equilibrium states and the ergodic theory of Anosov diffeomorphisms. Lecture Notes in Mathematics, Vol. 470. Berlin, Heidelberg, New York: Springer

3. Bowen, R., Ruelle, D.: The ergodic theory of axiom A flows. Invent. Math. 29, 181-202

4. Eckmann, J.P., Ruelle, D.: Ergodic theory of chaos and strange attractors. Rev. Mod. Phys. 57, 617-656

5. Ellis, R.S.: Lage deviations for a general class of random vectors. Ann. Probab. 12, 1-12

6. Hille, E.: Analytic function theory. Aylesbury: Ginn

7. Katok, A.: Lyapunov exponents, entropy and periodic orbits for diffeomorphisms. Publ. Math. I.H.E.S. 51, 137-174

8. Mañé, R.: The Hausdorff Dimension of invariant probabilities of rational maps. Lecture Notes in Mathematics, Vol. 1331, pp. 86-117. Berlin, Heidelberg, New York: Springer

9. Martens, M., de Melo, W., van Strien, S.: Julia Fatou Sullivan theory for real one-dimensional dynamics, Preprint Delft

10. de Melo, W.: "Lectures on one dimensional dynamics," $17^{\circ}$ Coloquio Brasileiro de Matemática. IMPA

11. Newhouse, S.: Continuity properties of entropy. Ann. Math. 129, 215-235

12. Pelikan, S.: The duration of transients. Trans. Am. Math. Soc. 287, 215-221

13. Walters, P.: An introduction to ergodic theory. Graduate Texts in Math. Berlin, Heidelberg, New York: Springer

14. Takahashi, Y.: Entropy functional (free energy) for dynamical systems and their random perturbations. Proc. Taniguchi. Symp. on stochastic analysis. Katata and Kyoto. 1980. Kinokuniga, North-Holland

15. Yomdim, Y.: Volume growth and entropy. Israel J. Math. 57-3, 285-300

Communicated by J.-P. Eckmann 
